\title{
Effect of 42 amino acid long amyloid- $\beta$ peptides on Arabidopsis plants
}

\author{
HanGyeol Lee $\cdot$ Ji Woo Kim $\cdot$ Sangyun Jeong $\cdot$ Jungeun An $\cdot$ Young-Cheon Kim $\cdot$ Hojin Ryu $\cdot$ Jeong Hwan Lee
}

Received: 21 October 2020 / Revised: 3 November 2020 / Accepted: 4 November 2020

(c) Korean Society for Plant Biotechnology

\begin{abstract}
Although the evolution of Arabidopsis thaliana and humans diverged approximately 1.6 billion years ago, recent studies have demonstrated that protein function and cellular processes involved in disease response remain remarkably conserved. Particularly, $\gamma$-secretase, a multisubunit protein complex that participates in intramembrane proteolysis (RIP) regulation, is also known to mediate the cleavage of more than 80 substrates including the amyloid precursor protein (APP) and the Notch receptor. Although the genes (PS1/2, APH-1, PEN-2, and NCT) coding for the $\gamma$-secretase complex components are present in plant genomes, their function remains largely uncharacterized. Given that the deposition of 42 amino acid long amyloid- $\beta$ peptides $\left(\mathrm{hA} \beta_{42}\right)$ is thought to be one of the main causes of Alzheimer's disease, we aimed to examine the physiological effects of $\mathrm{hA} \beta_{42}$ peptides on plants. Interestingly, we found that Arabidopsis protoplast death increased after $24 \mathrm{~h}$ of exposure to 3 or $5 \mu \mathrm{M} \mathrm{hA} \beta_{42}$ peptides. Furthermore, transgenic Arabidopsis plants overexpressing the $h A \beta_{42}$ gene exhibited changes in primary root length and silique phyllotaxy . Taken together, our results demonstrate that hA $\beta_{42}$ peptides, a metazoan protein, significantly affect Arabidopsis protoplast viability and plant morphology.
\end{abstract}

Keywords Alzheimer's disease, Amyloid- $\beta$ peptide, Arabidopsis mesophyll protoplasts, $\gamma$-secretase, Transgenic plants

\section{Introduction}

Large functional genomics initiatives have revealed that many

H. G. Lee · J. W. Kim • S. Jeong • J. An • Y.-C. Kim • J. H. Lee (ه) Division of Life Sciences, Jeonbuk National University, 567 Baekje-daero, Deokjin-gu, Jeonju, 54896, Republic of Korea e-mail: jhwanlee90@jbnu.ac.kr

Hojin Ryu $(\varangle)$

Department of Biology, Chungbuk National University, Cheongju 28644, Republic of Korea

e-mail: hjryu96@chungbuk.ac.kr human disorders are affected by multiple genes and their genetic and molecular interactions with each other and the environment. Thus, the use of various model systems such as human and mammalian cell lines, yeast (i.e., Saccharomyces cerevisiae), as well as other organisms such as Caenorhabditis elegans and Drosophila melanogaster contributes to important discoveries within human disease research and the design of new diagnostic tools and medical treatment strategies (Xu and Moller 2011).

Recent reports have shown that Arabidopsis thaliana known as a model organism for plant biology is a valuable model system to study molecular mechanisms underlying human disease states (Hays 2002). According to the comparison between Arabidopsis and human genome sequences, Arabidopsis genome encodes many orthologues of human proteins albeit Arabidopsis and humans diverged 1.6 billion years ago (Xu and Moller 2011). For example, TAIR WU-BLAS tool (http://www.arabidopsis.org) analysis showed that $71 \%$ of genes involved in neurological human diseases have Arabidopsis orthologues with high $E$-value cutoffs. Furthermore, Arabidopsis has some advantages for human disease research: (i) Simple cultivation and maintenance with low infrastructure and operating costs, (ii) Fast and simple transformation techniques for transgenic analyses, (iii) The availability of large mutants and genomic resources, and (iv) few ethical restrictions (Xu and Moller 2010).

Alzheimer's disease $(\mathrm{AD})$ is the most common age-related neurodegenerative disorder of the central nervous system (Mattson 2004), which is characterized by the deposition of aggregation-prone amyloid- $\beta$ peptides $(A \beta s)$ as the degraded products of the amyloid precursor protein (APP) and neurofibrillary tangles in the brain (Selkoe 1998). In humans, presenilin (PS) was identified from the genetic screens of patients suffering from $\mathrm{AD}$ and mutations in PS1 or PS2 led to improper cleavage of APP, which resulting in $A \beta s$ accumulation and progressive neurodegeneration (Sherrington et al. 1995). PS as a catalytic core protein is a component of $\gamma$-secretase, which is implicated in the process 
of regulated intramembrane proteolysis (RIP) (Sannerud and Annaert 2009). Besides PS, nicastrin (NCT), presenilin enhancer 2 (PEN-2), and anterior pharynx-defective 1 (APH-1) as other constituents of $\gamma$-secretase are required for complex assembly, stabilization, trafficking, and substrate recognition (Parks and Curtis 2007).

APP as one of more than 80 substrates for $\gamma$-secretase is a metazoan protein, which is only present in genomes of multicellular animals (Haapasalo and Kovacs 2011). However, the components of $\gamma$-secretase have been identified in both metazoan and plants (Grigorenko et al. 2002; Kimberly and Wolfe 2003; Moliaka et al. 2004; Ponting et al. 2002; Wigge and Weigel 2001). The moss Physcomitrella patens also harbors homologues of all $\gamma$-secretase subunits with $P S$ present in one copy. A lesion in moss $P S$ (PPPS) showed several pleiotropic defects such as abnormal growth pattern, impaired chloroplast movement, and decreased uptake of an endocytosis tracer (Khandelwal et al. 2007). The mutation in PS of Dictyostelium discoideum as a slime mould affected cell fate determination and the regulation of phagocytosis (McMains et al. 2010). In Arabidopsis as a higher plant, all $\gamma$-secretase subunits interacted with each other and colocalized in endomembrane system compartments (Smolarkiewicz et al. 2014). These data suggest that $\gamma$-secretase is evolutionarily conserved and its role may be extended beyond RIP found from animal studies.

Here we presented the effect of 42 amino acids longhuman amyloid- $\beta$ peptide $\left(\mathrm{hA} \beta_{42}\right)$ on Arabidopsis protoplasts, tissues, and transgenic plants.

\section{Materials and Methods}

Plant materials and growth conditions

The wild-type [ecotype Columbia (Col-0)] and the transgenic Arabidopsis plants were grown in soil or a murashige and skoog (MS) medium at $23^{\circ} \mathrm{C}$ under long-day (LD) conditions (16 h light $/ 8 \mathrm{~h}$ dark) at a light intensity of 120 $\mathrm{mmol} \cdot \mathrm{m}^{-2} \cdot \mathrm{s}^{-1}$.

For treatment of 42 amino acids long-human amyloid- $\beta$ peptide $\left(\mathrm{hA} \beta_{42}\right)$ at cellular level, we followed the procedures for isolation of Arabidopsis mesophyll protoplasts, as previously described (Lee et al. 2017; Yoo et al. 2007). Isolated protoplasts $\left(2 \times 10^{4}\right)$ were treated with 3 and $5 \mathrm{mM} \mathrm{hA} \beta_{42}$ monomer or DMSO, and then the percentage of dead cells at indicated times was determined by staining protoplasts with Evans blue dye (Sigma-Aldrich), which was added to the samples to a final concentration of $0.04 \%$ (Danon et al. 2005; Wright et al. 2000). The number of stained cells was measured using a light microscope (Zeiss, Oberkochen, Germany). For treatment of $\mathrm{hA} \beta_{42}$ at tissue level, Arabidopsis plants were grown on soil or MS medium under LD conditions at $23^{\circ} \mathrm{C}$. 10 -d-old plants were treated with $3 \mathrm{mM}$ $\mathrm{hA} \beta_{42}$ monomer or Dimethyl sulfoxide (DMSO) (SigmaAldrich, St. Louis, Missouri, USA), as previously described (Kim et al. 2017), and then $h A \beta_{42}$-treated plants were observed after 2 and 4 days under a light microscope (Nikon, Tokyo, Japan).

\section{Preparation of hAß42 monomer}

The procedures for were previously described (Dahlgren et al. 2002; Kook et al. 2012). hA $\beta_{42}$ peptide was dissolved at a concentration of $1 \mathrm{mg} \cdot \mathrm{ml}^{-1}$ in 3,3,3-Hexafluoro-2propanol (HFIP) (Sigma-Aldrich). HFIP was evaporated using Speed Vacuum concentrator and lyophilized peptide was resolved in $10 \mathrm{ml}$ DMSO. After calculation of the molar concentration, aliquots of $1 \mathrm{mM}$ lyophilized $\mathrm{hA} \beta_{42}$ monomer were stored at $-80^{\circ} \mathrm{C}$ until use.

Generation of transgenic Arabidopsis plants

For $p 35 S:: h A \beta_{42}$ construct, the region of human $A \beta_{42}$ was polymerase chain reaction (PCR)-amplified using plasmid DNA template with the gene encoding human amyloid precursor protein (hAPP) [gifted form Dr. Jeong (Jeonbuk National University, South Korea) and the resulting products were cloned into the pCHF3 vector harboring cauliflower mosaic virus (CaMV) $35 \mathrm{~S}$ promoter. The resulting recombinant plasmid was sequenced to verify the absence of the PCR errors during amplification. Oligonucleotide primers used for cloning were as follows: 5'-ACGAGCTC GGTACCCGGGATGCTGCCCGGTTTGGC -3' and 5'-CT CTAGAGGATCCCCGGGCGCTATGACAACACCGCCC -3'. This construct was transformed into Agrobacterium tumefaciens strain GV3101 and then infiltrated into the wild-type (Col-0) plants. Transgenic plants were generated using the floral dip method with minor modifications (Weigel and Glazebrook 2002). The transgenic seedlings were first selected using kanamycin for a $\mathrm{pCHF} 3$ vector and then verified using PCR-based DNA genotyping. At least 30 40 $\mathrm{T}_{1}$ seedlings were analyzed for the construct.

To examine the expression of $h A \beta_{42}$ in the transgenic Arabidopsis plants, we performed semi-quantitative reverse transcription (RT)-PCR, as described previously (Lee et al. 2007). Total RNA was extracted from 8-d-old seedlings using a RiboEx Total RNA kit (GeneAll, Seoul, Korea), and 
first-strand complementary DNA (cDNA) was synthesized from $1 \mu \mathrm{g}$ of total RNA, in accordance with the manufacturer's instructions (GeneAll). The following oligonucleotides were employed as gene-specific primers in the RT-PCR analyses: for $h A \beta_{42}, 5$ '-GAGCTCATGCTGCCCGGTTTGG CACT -3' and 5'-TCTAGACGCTATGACAACACCGCCC ACC-3'; for AtPP2AA3, 5'-GCGGTTGTGGAGAACATGA TACG-3' and 5'-GAACCAAACACAATTCGTTGCTG-3'. $A t P P 2 A A 3$ was used as a reference gene.

\section{Results and Discussion}

The treatment of hA $\beta_{42}$ monomer in Arabidopsis mesophyll protoplasts affects the viability of the cells

Because the accumulation of aggregated form of $A \beta_{42}$ in the brain leads to mitochondrial dysfunction and elevated reactive oxygen species (ROS), consequently resulting in cell death (Reddy and Beal 2008), we investigated whether $\mathrm{hA} \beta_{42}$ monomer affects the viability of plant cells. Mesophyll protoplasts were isolated form 10-d-old Arabidopsis wild-type (Col-0) plants grown at $23^{\circ} \mathrm{C}$ under $\mathrm{LD}$ conditions and $2 \times$ $10^{4}$ protoplasts were treated with 3 or $5 \mathrm{mM} \mathrm{hA} \beta_{42}$ monomer at $1,2,4,8$, and $24 \mathrm{~h}$ under continuous light conditions. The protoplasts treated with DMSO were used as a control. Because dead cells were specifically stained with Evans blue dye (Danon et al. 2005; Wright et al. 2000), we stained 3 or $5 \mathrm{mM} \mathrm{hA} \beta_{42}$ monomer-treated protoplasts after $24 \mathrm{~h}$ with Evans blue dye. This indicates that dead cells were increased in the protoplasts treated with 3 or $5 \mathrm{mM} \mathrm{hA} \beta_{42}$ monomer, compared with DMSO-incubated protoplasts (Fig. 1a). We also calculated the percentage of dead cells at $0,1,2,4,8$, and $24 \mathrm{~h}$ under a light microscope. The percentage of dead cells in protoplasts treated with hA $\beta_{42}$ monomer gradually increased and reached approximately $60 \%$ after $24 \mathrm{~h}$ (Fig. 1b). The portion of dead cells was nearly similar irrespective of treatment of 3 or $5 \mathrm{mM} \mathrm{hA} \beta$ 42 monomer. In contrast, the percentage of dead cells in DMSO-treated mesophyll protoplasts remained almost constant until $8 \mathrm{~h}$ and showed approximately $40 \%$ after $24 \mathrm{~h}$ (Fig. 1b). These results indicated that $\mathrm{hA} \beta_{42}$ monomer reduces the viability of Arabidopsis mesophyll protoplasts.

The treatment of $\mathrm{hA} \beta_{42}$ monomer does not cause any morphological changes in Arabidopsis young seedlings

Because $\mathrm{hA} \beta_{42}$ monomer affects the viability of Arabidopsis mesophyll protoplasts at cellular level (Fig. 1), we next exam- (a)
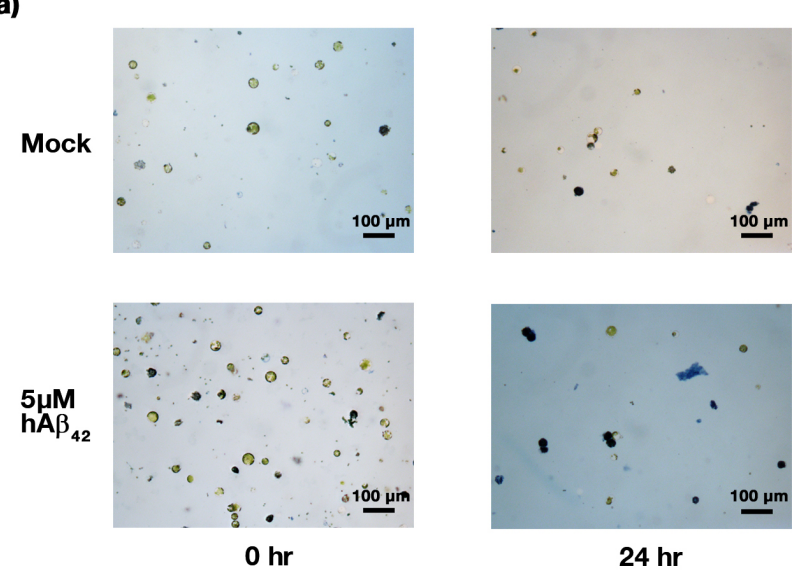

(b)

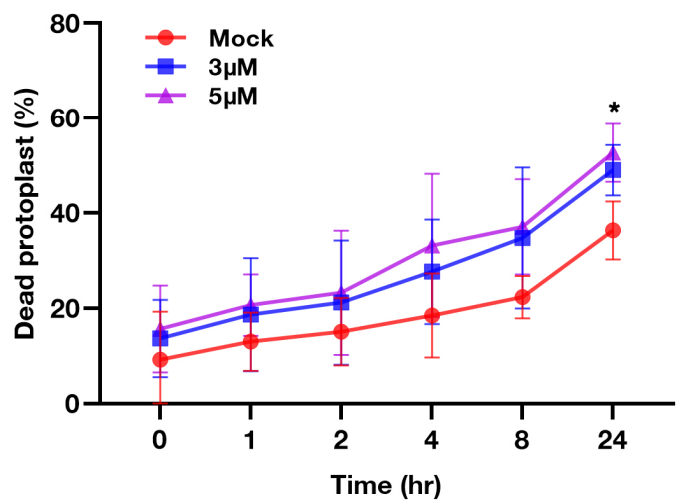

Fig. 1 Effect of $\mathrm{hA} \beta_{42}$ peptide treatment on Arabidopsis mesophyll protoplasts. (a) Morphology of protoplasts treated with $5 \mu \mathrm{M}$ of hA $\beta_{42}$ monomer and DMSO. 10-d-old Arabidopsis (Col-0) plants were used for isolation of protoplasts, after which $2 \times 10^{4}$ protoplasts were incubated at $23^{\circ} \mathrm{C}$ under continuous light conditions and treated with 3 and $5 \mu \mathrm{M}$ of $\mathrm{hA} \beta_{42}$ monomer. After staining protoplasts with Evans blue dye, the dead cells were observed under the microscope (scale bars: $100 \mu \mathrm{m}$ ). (b) Percentage of dead cells at $0,1,2,4,8$, and 24 hours. The asterisks indicate a significant difference in the percentage of dead cells in 3 and $5 \mu \mathrm{M} \mathrm{hA} \beta_{42}$ monomer-treated protoplasts compared with DMSOtreated protoplasts (Student's t-test, ${ }^{*} P<0.05$ )

ined the effect of $\mathrm{hA} \beta_{42}$ monomer at plant tissue levels. Then, we sowed Arabidopsis wild-type (Col-0) seeds in solid MS medium, transferred 10-d-old seedlings into 6-well plates containing $1 \mathrm{~mL}$ of liquid MS medium with $3 \mathrm{mM}$ $\mathrm{hA} \beta_{42}$ monomer or DMSO, and observed the the morphological changes of seedlings after 2 and 4 days. The $3 \mathrm{mM}$ $\mathrm{hA} \beta_{42}$ monomer-treated seedlings after 2 and 4 days did not show any defects of morphology such as leaves, hypocotyls, and roots (Fig. 2). This result indicated that $\mathrm{hA} \beta_{42}$ monomer does not affect the growth of young seedlings at the tissue level. However, we cannot dismiss the possibility that young seedlings may be more resistant to physical and chemical treatment than protoplasts. 
$3 \mu \mathrm{M} \mathrm{hA} \beta_{42}$
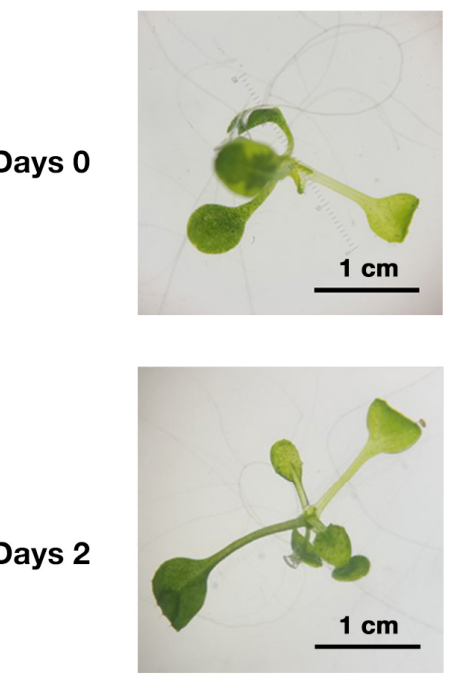

Days 2

Days 0

Days 4

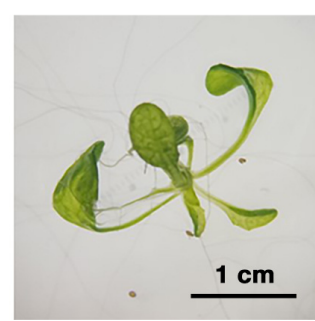

DMSO
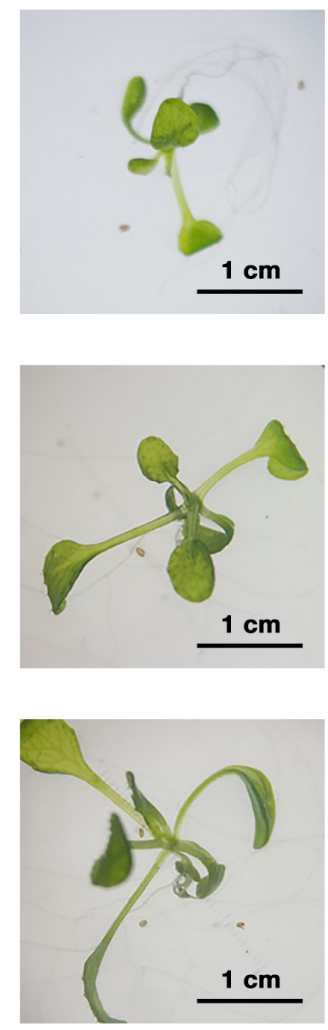

Fig. 2 Effect of A $\beta 42$ peptide treatment on Arabidopsis young seedlings. 10-d-old wild-type Arabidopsis (Col-0) plants grown at $23^{\circ} \mathrm{C}$ under long-day (LD) conditions were treated with $3 \mu \mathrm{M}$ $\mathrm{hA} \beta_{42}$ monomer and morphological changes in Arabidopsis plants were observed after 2 and 4 days. DMSO-treated Arabidopsis seedlings were used as controls

The overexpression of $\mathrm{hA} \beta_{42}$ in transgenic Arabidopsis plants leads to morphological changes in root length and silique phyllotaxy

In order to investigate the effect of the $\mathrm{hA} \beta_{42}$ overexpression in Arabidopsis plants, we expressed the region of $h A \beta_{42}$ under the control of a $35 S$ promoter $\left(p 35 S:: h A \beta_{42}\right)$ in Arabidopsis wild-type (Col-0) plants. We obtained 37 independent transgenic plants in the $\mathrm{T}_{1}$ generation from DNA genotyping (Fig. 3a). To examine the morphological changes of the $p 35 S:: h A \beta_{42}$ plants in more detail, we generated the transgenic plants with a single $p 35 S:: h A \beta_{42}$ insertion in the $\mathrm{T}_{3}$ generation and confirmed a higher expression level of introduced $h A \beta_{42}$ gene (Fig. 3b). Firstly, we compared root growth phenotypes of wild-type (Col- 0$)$ and $p 35 S:: h A \beta_{42}$ plants. The seedlings were grown on MS medium and primary root lengths were quantified 8 days after germination. We found that three independent $p 35 S:: h A \beta_{42}$ lines had long primary roots than wild-type (Col-0) seedlings (Fig. 3c). The degree of primary root lengths was correlated with the expression levels of $h A \beta_{42}$ gene in independent $p 35 S:: h A \beta_{42}$ lines. For instance, a $p 35 S:: h A \beta_{42}$ line (\#16) showing strong $h A \beta_{42}$ gene expression had more longer primary roots than $p 35 S:: h A \beta_{42}$ line (\#8). Secondly, we measured the flowering time in six independent $p 35 S:: h A \beta_{42}$ lines under long-day (LD) conditions at $23^{\circ} \mathrm{C}$. We found that the flowering time of four independent $p 35 S:: h A \beta_{42}$ lines (\#14, \#22, \#26, and \#36) was unchanged, whereas two independent lines (\#8 and \#16) has altered flowering time (Fig. $3 \mathrm{~d}$ and e). However, the degree of flowering time was not correlated with the expression levels of $h A \beta_{42}$ gene in independent $p 35 S:: h A \beta_{42}$ lines. Lastly, we detected abnormal phyllotaxy in the inflorescence of two independent $p 35 S:: h A \beta_{42}$ lines (\#16 and \#22) (Fig. 3f), which showing that strong $h A \beta_{42}$ gene expression in $p 35 S:: h A \beta_{42}$ lines affected the degree of defects in phyllotaxy. These results indicated that the increased $h A \beta_{42}$ expression affects morphological changes in root length and silique phyllotaxy in the $p 35 S:: h A \beta_{42}$ plants.

\section{Conclusions}

Here we provide the first data on the effect of $h A \beta_{42}$ monomer as a metazoan protein in angiosperms. In a series of experiments, we showed that the viability of cells was reduced in $\mathrm{hA} \beta_{42}$ monomer-treated Arabidopsis mesophyll protoplasts (Fig. 1), suggesting that $\mathrm{h} A \beta_{42}$ monomer may lead to cell death. Because the protoplast system is known as a useful tool that allows an easy uptake of bioactive agents and a concise quantification of cell death response (Yoo et al. 2007), we will further investigate whether the features of the cellular destruction such as DNA cleavage, the activation of caspase-like molecules, and the release of mitochondrial components are observed in $\mathrm{hA} \beta_{42}$ monomer-treated protoplasts (Reape and McCabe 2008). Although treatment of $\mathrm{hA} \beta_{42}$ monomer did not affect the growth of young seedlings at the tissue level, $h A \beta_{42}$ gene expression in the transgenic Arabidopsis plants caused the morphological changes in primary root length and silique phyllotaxy (Fig. 3). A recent report have shown that Arabidopsis ps1 ps2 double mutants have accelerated chlorosis in the dark (Smolarkiewicz et al. 2014). Because darkness and starvation induce senescence and autophagy in plants (Sobieszczuk-Nowicka et al. 2018), we will further examine the effect of prolonged darkness treatment on $\mathrm{hA} \beta_{42}$ monomer-treated Arabidopsis seedlings and $p 35 S:: h A \beta_{42}$ plants. 
(a)

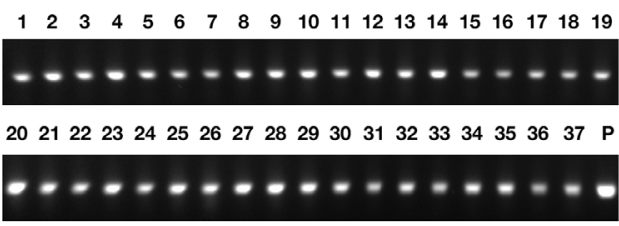

(c)

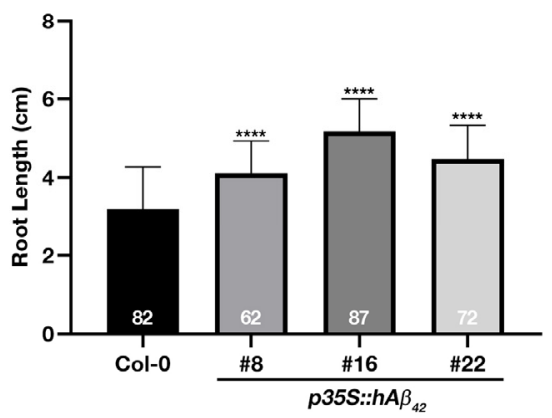

(e)

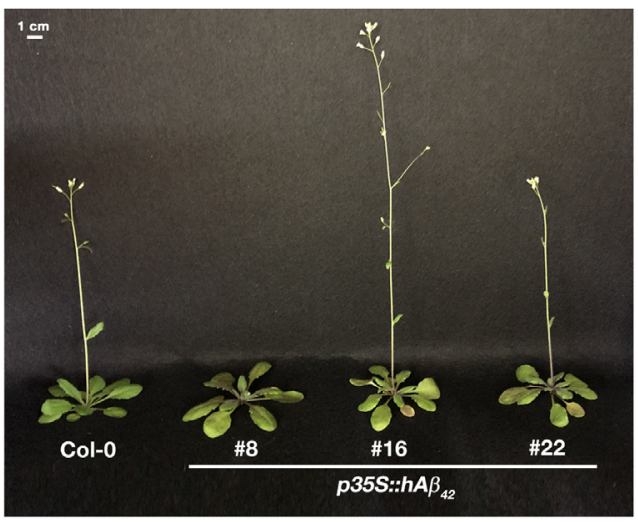

(b)

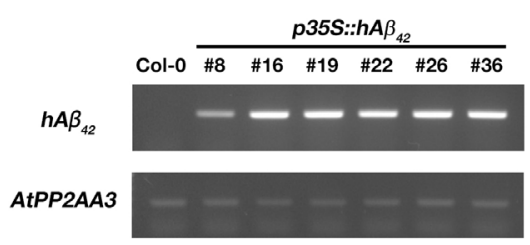

(d)

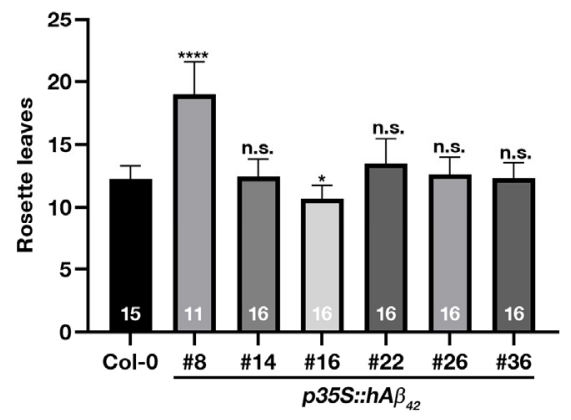

(f)

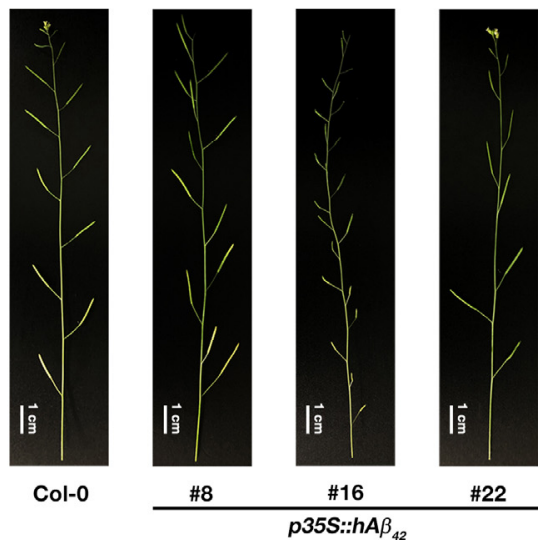

Fig. 3 Effect of $h A \beta_{42}$ overexpression on transgenic Arabidopsis plants. (a) Confirmation of $h A \beta_{42}$ integration in $p 35 S:: h A \beta_{42}$ plants at the $\mathrm{T}_{3}$ generation. $\mathrm{P}$ was used as a control for DNA genotyping. (b) Overexpression of $h A \beta_{42}$ in $p 35 S:: h A \beta_{42}$ plants at the $\mathrm{T}_{3}$ generation. Wild-type Arabidopsis (Col-0) plant was used as a control for RT-PCR. (c) Root length measurements in $p 35 S: \because h A \beta_{42}$ plants. The number of measured plants is shown above each genotype in the bar graph. The error bars indicate the standard error of the mean of three biological replicates. Asterisks denote statistically significant differences compared with wild-type Arabidopsis (Col-0) plants (Student's $t$-test, ${ }^{* * *} P<0.001$ ). (d and e) Flowering time phenotypes of $p 35 S: \because h A \beta_{42}$ plants at $23^{\circ} \mathrm{C}$ under LD conditions. Asterisks denote statistically significant differences compared with wild-type Arabidopsis (Col-0) plants (Student's $t$-test, ${ }^{*} P<0.05,{ }^{* * *} P<0.001$, n.s., not significant). Photographs were taken when wild-type Arabidopsis (Col-0) plants flowered. Scale bars: $1 \mathrm{~cm}$. (f) Silique phyllotaxy in $p 35 S:: h A \beta_{42}$ plants. Scale bars: $1 \mathrm{~cm}$

\section{Acknowledgements}

We thank Dr. Mook-Jung (Seoul National University) for kindly providing research methods. This work was supported by National University Promotion Program in 2019 (to J.H. Lee).

\section{References}

Dahlgren KN, Manelli AM, Stine WB, Jr., Baker LK, Krafft GA,
LaDu MJ (2002) Oligomeric and fibrillar species of amyloid-beta peptides differentially affect neuronal viability. J Biol Chem 277:32046-32053

Danon A, Miersch O, Felix G, Camp RG, Apel K (2005) Concurrent activation of cell death-regulating signaling pathways by singlet oxygen in Arabidopsis thaliana. Plant J 41:68-80

Grigorenko AP, Moliaka YK, Korovaitseva GI, Rogaev EI (2002) Novel class of polytopic proteins with domains associated with putative protease activity. Biochemistry (Mosc) 67:826-835

Haapasalo A, Kovacs DM(2011) The many substrates of presenilin/ gamma-secretase. J Alzheimers Dis 25:3-28

Hays JB (2002) Arabidopsis thaliana, a versatile model system for 
study of eukaryotic genome-maintenance functions. DNA Repair (Amst) 1:579-600

Khandelwal A, Chandu D, Roe CM, Kopan R, Quatrano RS (2007) Moonlighting activity of presenilin in plants is independent of gamma-secretase and evolutionarily conserved. Proc Natl Acad Sci U S A 104:13337-13342

Kim HJ, Wu CY, Yu HM, Sheen J, Lee H(2017) Dual CLAVATA3 peptides in Arabidopsis shoot stem cell signaling. J Plant Biol 60:506-512

Kimberly WT, Wolfe MS (2003) Identity and function of gammasecretase. J Neurosci Res 74:353-360

Kook SY, Hong HS, Moon M, Ha CM, Chang S, Mook-Jung I (2012) $\operatorname{Abeta}(1)(-)(4)(2)-R A G E$ interaction disrupts tight junctions of the blood-brain barrier via $\mathrm{Ca}(2)(+)$-calcineurin signaling. $\mathrm{J}$ Neurosci 32:8845-8854

Lee JH, Jin S, Kim SY, Kim W, Ahn JH (2017) A fast, efficient chromatin immunoprecipitation method for studying protein-DNA binding in Arabidopsis mesophyll protoplasts. Plant Methods 13:42

Lee JH, Park SH, Lee JS, Ahn JH(2007) A conserved role of SHORT VEGETATIVE PHASE (SVP) in controlling flowering time of Brassica plants. Biochim Biophys Acta 1769:455-461

Mattson MP (2004) Pathways towards and away from Alzheimer's disease. Nature 430:631-639

McMains VC, Myre M, Kreppel L, Kimmel AR(2010) Dictyostelium possesses highly diverged presenilin/gamma-secretase that regulates growth and cell-fate specification and can accurately process human APP: a system for functional studies of the presenilin/gamma-secretase complex. Dis Model Mech 3:581-594

Moliaka YK, Grigorenko A, Madera D, Rogaev EI (2004) Impas 1 possesses endoproteolytic activity against multipass membrane protein substrate cleaving the presenilin 1 holoprotein. FEBS Lett 557:185-192

Parks AL, Curtis D (2007) Presenilin diversifies its portfolio. Trends Genet 23:140-150

Ponting CP, Hutton M, Nyborg A, Baker M, Jansen K, Golde TE (2002) Identification of a novel family of presenilin homologues. Hum Mol Genet 11:1037-1044

Reape TJ, McCabe PF (2008) Apoptotic-like programmed cell death in plants. New Phytol 180:13-26

Reddy PH, Beal MF (2008) Amyloid beta, mitochondrial dysfunction and synaptic damage: implications for cognitive decline in aging and Alzheimer's disease. Trends Mol Med 14:45-53

Sannerud R, Annaert W (2009) Trafficking, a key player in regulated intramembrane proteolysis. Semin Cell Dev Biol 20:183-190

Selkoe DJ (1998) The cell biology of beta-amyloid precursor protein and presenilin in Alzheimer's disease. Trends Cell Biol 8:447-453

Sherrington R, Rogaev EI, Liang Y, Rogaeva EA, Levesque G, Ikeda M, Chi H, Lin C, Li G, Holman K, Tsuda T, Mar L, Foncin JF, Bruni AC, Montesi MP, Sorbi S, Rainero I, Pinessi L, Nee L, Chumakov I, Pollen D, Brookes A, Sanseau P, Polinsky RJ, Wasco W, Da Silva HA, Haines JL, Perkicak-Vance MA, Tanzi RE, Roses AD, Fraser PE, Rommens JM, St George-Hyslop PH (1995) Cloning of a gene bearing missense mutations in earlyonset familial Alzheimer's disease. Nature 375:754-760

Smolarkiewicz M, Skrzypczak T, Michalak M, Lesniewicz K, Walker JR, Ingram G, Wojtaszek P (2014) Gamma-secretase subunits associate in intracellular membrane compartments in Arabidopsis thaliana. J Exp Bot 65:3015-3027

Sobieszczuk-Nowicka E, Wrzesinski T, Bagniewska-Zadworna A, Kubala S, Rucinska-Sobkowiak R, Polcyn W, Misztal L, Mattoo AK (2018) Physio-genetic dissection of dark-induced leaf senescence and Tyiming its reversal in barley. Plant Physiol 178: 654-671

Weigel D, Glazebrook J (2002) Arabidopsis: A laboratory manual. Cold Spring Harbor Laboratory Press, Cold Spring Harbor, N.Y.

Wigge PA, Weigel D (2001) Arabidopsis genome: life without notch. Curr Biol 11:R112-114

Wright KM, Duncan GH, Pradel KS, Carr F, Wood S, Oparka KJ, Cruz SS (2000) Analysis of the N gene hypersensitive response induced by a fluorescently tagged tobacco mosaic virus. Plant Physiol 123:1375-1386

Xu XM, Moller SG (2010) ROS removal by DJ-1: Arabidopsis as a new model to understand Parkinson's Disease. Plant Signal Behav 5:1034-1036

Xu XM, Moller SG (2011) The value of Arabidopsis research in understanding human disease states. Curr Opin Biotechnol 22:300-307

Yoo SD, Cho YH, Sheen J (2007) Arabidopsis mesophyll protoplasts: a versatile cell system for transient gene expression analysis. Nat Protoc 2:1565-1572 